\title{
Clinical Features and Long-term Survivals of Metastatic Hepatic Neuroendocrine Neoplasms Secondary to Gastro-entero-pancreatic Site: An Analysis by Applying the World Health Organization 2017 Grading Classification
}

\author{
Min Yang \\ West China Hospital of Sichuan University \\ Lin Zeng \\ West China Hospital of Sichuan University \\ Sheng-zhong Hou \\ West China Hospital of Sichuan University \\ Neng-wen Ke \\ West China Hospital of Sichuan University \\ Bo-le Tian \\ West China Hospital of Sichuan University \\ Xu-bao Liu \\ West China Hospital of Sichuan University \\ Bo Xiang \\ West China Hospital of Sichuan University \\ Yi Zhang ( $\square$ zhangyide520@163.com ) \\ West China Hospital of Sichuan University https://orcid.org/0000-0003-3641-0629
}

\section{Research article}

Keywords: Neuroendocrine neoplasms, Hepatic, Metastasis, WHO, Grading, Prognosis

Posted Date: July 24th, 2020

DOI: https://doi.org/10.21203/rs.3.rs-46070/v1

License: (c) (i) This work is licensed under a Creative Commons Attribution 4.0 International License.

Read Full License 


\section{Abstract \\ Background and Purpose}

Hepatic neuroendocrine neoplasms (NENs) are very rare, in which metastatic hepatic NENs (MH-NENs) secondary to gastro-entero-pancreatic NENs (GEP-NENs) account for their majority. In 2017, World Health Organization (WHO) redefined GEP-NENs into G1 neuroendocrine tumors (NETs), G2 NETs and G3 NETs and G3 neuroendocrine carcinomas (G3 NECs), which has not been rigorously validated for MH-NENs secondary to GEP-NENs.

\section{Method:}

Data of patients who were surgically treated and clinicopathologically diagnosed as MH-NENs secondary to GEP-NENs at West China Hospital of Sichuan University from January 2006 to December 2018 were retrospectively analyzed by applying the WHO 2017 grading classification.

\section{Results}

We identified 150 patients with MH-NENs secondary to GEP-NENs, including 10 patients with WHO 2017 G1 NETs, 26 with G2 NETs, 33 with G3 NETs and 81 with G3 NECs. The estimated 3-year overall survival for each new grading group was $100 \%, 79.4 \%, 49.5 \%$ and $20.7 \%$, respectively. Survival of G1 NETs or G2 NETs was significantly better than that of G3 NETs $(P=0.013, P=0.037$; respectively) and G3 NECs ( $P=$ $0.001, P<0.001$; respectively). Patients with G3 NECs present notably worse survival than those with $G 3$ NETs $(P=0.012)$, while survival comparison between G1 NETs and G2 NETs wasn't statistically different $(P=0.131)$. The WHO 2017 grading classification was effective independent predictor of survival for $\mathrm{MH}-$ NENs secondary to GEP-NENs (hazard ratio: $4.234 ; 95 \%$ confidence intervals: $1.984-6.763 ; P=0.003$ ).

\section{Conclusion}

Our demonstration revealed that the WHO 2017 grading classification could well stratify MH-NENs secondary to GEP-NENs into prognostic groups and supported its wide use in clinical practice.

\section{Introduction}

Neuroendocrine neoplasms (NENs) are a group of amine precursor uptake and decarboxylation neoplasms which are highly heterogeneous with distinct pathological features, biological behaviors and prognosis ${ }^{[1-3]}$. NENs could occur in various organs or tissues with neuroendocrine cells, which might secrete bioactive amines or peptide hormones, such as insulin, gastrin, prostaglandins, serotonin, etc. and present different clinical manifestations ${ }^{[1-3]}$. NENs arising from the digestive tract account for the 
highest rate of incidence of NENs, in which gastro-entero-pancreatic neuroendocrine neoplasms (GEPNENs) are the most common (about 65\%-67\%), followed by those from bronchopulmonary system (about $27 \%)^{[3-5]}$. Studies have indicated the incidence of NENs has increased obviously in the past decades, probably due to the development of endoscopic and radiological imaging techniques, the improvement of clinical awareness and modified diagnostic techniques for NENs ${ }^{[5-7]}$.

NENs often grow to an advanced stage with either locally unresectable primary tumors or distant metastasis on admission, due to the atypical or unobvious clinical manifestations which are usually difficult to be discovered in early days ${ }^{[5-8]}$. With the unique anatomical characteristics, liver is the most common site of metastasis for NENs, especially for GEP-NENs. It has been reported that nearly $80 \%$ of hepatic neuroendocrine neoplasms (H-NENs) are metastatic with synchronous or metachronous masses in primary endocrine sites ${ }^{[6,9]}$, while primary hepatic neuroendocrine neoplasms (PH-NENs) are extremely rare, accounting only for $0.3 \%-4 \%$ of all NENs and $0.28 \%-0.46 \%$ of all hepatic malignancies ${ }^{[7,10]}$. Also, metastatic hepatic neuroendocrine neoplasms (MH-NENs) may be the first manifestation in up to 40$90 \%$ of cases, which decrease the survival of NENs in varying degrees ${ }^{[11,12]}$. With the significantly rising incidence of NENs in the past years ${ }^{[5-7]}$, there is still few available data on the clinical features and longterm survivals of MH-NENs secondary to GEP-NENs.

On the other hand, World Health Organization (WHO) histologically classified GEP-NENs into three main groups in 2010 based on the cut-off point of mitotic rate and Ki-67 proliferative index: G1 neuroendocrine tumors (NETs), G2 NETs and G3 neuroendocrine carcinoma ("G3 NECs") ${ }^{[13]}$. This grading classification for GEP-NENs derived from the one proposed by European Neuroendocrine Tumor Society in 2006 [14], which was recently proven to be a little out-of-date [15-17], although it was widely used thereafter ${ }^{[18-21]}$. In 2017, based on both morphological differentiation and grading upon proliferation rate, WHO updated its grading system which redefined GEP-NENs into 2 main categories and 4 groups: well-differentiated tumors (i.e. G1/G2/G3 NETs) and poorly-differentiated carcinomas (i.e. G3 NECs) ${ }^{\text {[22] }}$. However, this new WHO 2017 grading classification has not been rigorously validated before. Whether it is practical for grouping patients and predicting prognosis of MH-NENs secondary to GEP-NENs is still unclear.

In this study, based on the relevant data from a large Chinese single institute, we reviewed our experience with MH-NENs secondary to GEP-NENs, with the primary goal of evaluating the prognostic relevance of the novel WHO 2017 grading classification. To accomplish this, we summarized and compared the clinicopathological features and long-term survivals of MH-NENs secondary to GEP-NENs between each new grading group. This analysis represented, as far as we could know, the first attempt to do such a work.

\section{Materials And Methods Patients Enrollment}


Our research was approved by the institutional review board of West China Hospital of Sichuan University and written informed consent was obtained on admission from all patients, in accordance with the general principles of the Helsinki Declaration ${ }^{[23]}$. Patients who were both clinically and pathologically diagnosed as MH-NENs secondary to GEP-NENs at West China Hospital of Sichuan University from January 2006 to December 2018 were systematically reviewed from their electronic or paper-based

medical records, as we ever did in our previous studies ${ }^{[24,25]}$. Patients with a clinical suspicion of H-NENs but without a pathological identification weren't enrolled in this study. Patients with MH-NENs clinically originated from other organs or tissues, such as lung, were also excluded. Patients with PH-NENs were excluded as well. For included cases, data of sex, age, clinical presentation, imaging examination, surgical procedures, postoperative outcomes, and so on, were all retrospectively collected and analyzed.

\section{Tumors Feature}

The diagnosis of H-NENs was performed by surgical specimen or intraoperative biopsy from tumor tissues, which were routinely stained with hematoxylin-eosin and immunohistochemical methods and afterwards systematically reviewed by expert pathologists in our institution. The diagnosis of MH-NENs secondary to GEP-NENs was clinically based on the synchronous or metachronous pathological confirmation of NENs from gastro-entero-pancreatic site through the first or second operation. The histopathologic analyzing results (tumor size and location, major vessel invasion, lymph node invasion, portal vein thrombosis, extrahepatic invasion, surgical margin, morphological feature, differentiated degree, immunohistochemical staining examination for mitotic count, Ki-67 positive index, etc.) were all documented. In terms of the tumor grades, MH-NENs secondary to GEP-NENs in the present study were pathologically classified into 4 groups according to the new WHO 2017 grading classification for GEPNENs as follows: G1 NETs [ 2 mitoses per 10 high power fields (HPFs) and a Ki-67 proliferation index < $3 \%]$; G2 NETs (between 2 and 20 mitoses per 10 HPFs or a Ki-67 proliferation index ranging between $3 \%$ and $20 \%$ ); G3 NETs ( $>20$ mitoses per 10 HPFs or a Ki-67 proliferation index $>20 \%$ without poorlydifferentiated pathological features) and G3 NECs ( $>20$ mitoses per 10 HPFs or a Ki-67 proliferation index $>20 \%$ with poorly-differentiated small cell or large cell features) ${ }^{[22]}$.

\section{Statistical Analysis}

Follow-up was mainly conducted by telephone, e-mail or outpatient clinic review between June 2019 and January 2020 , leading to a median follow-up time of 38.2 mons (range 12.5-146.8mons). Overall survival (OS) was calculated as the number of months from the date of surgery (i.e. the date of diagnosis of liver metastatic disease for enrolled patients) to the date of last contact or time of death and presented as either median survival time (MST) or estimated 3-year OS with a hazard ratio (HR) or 95\% confidence intervals (Cls). Patients who were lost to follow-up were excluded in the final survival analysis models. Quantitative variables were reported as medians with ranges and compared by Student's t tests, while categorical variables were presented as numbers with frequencies or proportions (\%) and compared by Chi-square tests. OS was estimated using Kaplan-Meier (K-M) methods and compared using the log-rank 
test. Univariate and multivariate analyses were performed by Cox regression proportional hazards model to explore the potential prognosis-related factors for the OS of MH-NENs secondary to GEP-NENs.

Statistical analyses were performed using IBM SPSS version 22.0 software. A two-side $P$ value of $<0.05$ was considered statistically significant.

\section{Results}

\section{Patient demographics and clinicopathological characteristics}

According to the inclusive criteria of our present study, we identified 150 consecutive patients who were clinicopathologically diagnosed as MH-NENs secondary to GEP-NENs at West China Hospital of Sichuan University from January 2006 to December 2018 (Table 1). Our research consisted of 90 male patients and 60 females, with a median age of 56yrs. (range 42-74yrs.). Ninety-six MH-NENs were diagnosed synchronously with the primary lesions from gastro-entero-pancreatic site, while the rest of 54 metachronous cases were detected sometime after the first operation of GEP-NENs. There were 30, 12 and $44 \mathrm{MH}-\mathrm{NENs}$ which respectively originated from stomach, small intestine and large intestine, while 64 cases were secondary to pancreas. Eight-four patients with MH-NENs present nonspecific symptoms or signs related to hormone over-production, while 48 ones were detected incidentally by routine physical examination or postoperative review. One hundred and ten patients showed more than 1 visible hepatic lesions, with a median sum of the largest diameter of $13 \mathrm{~cm}$ (range $2-24 \mathrm{~cm}$ ). Multifocal lesions of 92 cases were located in both right and left half liver, while 127 patients present over 50\% estimated liver involvement. Fifty-seven and 30 patients were respectively treated by resection only and ablation only, while 41 ones were resected simultaneously with ablation during operation. The surgical margin of 57 MH-NENs was both grossly and microscopically negative (i.e. R0 resection). Ninety-three and 128 patients respectively received pre-and post-operative adjuvant therapy. There were respectively $57,65,27$ and 42 patients who showed regional lymph node metastases, portal vein tumor thrombus, vascular invasion and extrahepatic metastatic disease.

\section{Subgroup analysis according to the WHO 2017 grading classification}

Of 150 assessable patients with MH-NENs secondary to GEP-NENs, we defined 10 patients with G1 NETs, 26 with G2 NETs, 33 with G3 NETs and 81 with G3 NECs by the WHO 2017 grading classification (Table 1). There were distinct characteristics and clinicopathological differences between patients with G1/G2/G3 NETs and those with G3 NECs in our study. Notably, patients with G1/G2/G3 NETs were notably younger than those with G3 NECs $(P=0.041)$. G3 NECs present more synchronous liver lesion in comparison to $\mathrm{G} 1 / \mathrm{G} 2 / \mathrm{G} 3 \mathrm{NETs}(\mathrm{P}=0.032)$. G3 NECs were detected more incidentally than those with G1/G2/G3 NETs ( $P=0.014)$. The median sum of the largest diameter of all visible hepatic lesions with G1/G2/G3 NETs was significantly smaller than that of lesions with G3 NECs ( $P=0.047)$. G3 NECs present more vascular invasion and extrahepatic disease compared with those with $\mathrm{G} 1 / \mathrm{G} 2 / \mathrm{G} 3 \mathrm{NETs}(\mathrm{P}=0.017$, $\mathrm{P}=0.029$; respectively). It seemed that both $\mathrm{G} 1 / \mathrm{G} 2 / \mathrm{G} 3 \mathrm{NET}$ and $\mathrm{G} 3 \mathrm{NEC}$ sended to originate from large intestine and pancreas $(69.6 \%, 74.2 \%$; respectively), with a preferable metastatic location in right half liver 
(85.5\%, 91.3\%; respectively). Other comparisons between G1/G2/G3 NETs and G3 NECs, such as patients' sex, tumor type, multifocal lesions, estimated liver involvement, surgical procedure and margin, pre- and post-operative adjuvant therapy, regional lymph node metastases and portal vein tumor thrombus, weren't statistically significant $(P>0.05)$.

\section{Survival analysis for MH-NENs secondary to GEP-NENs}

When the follow-up ended in January 2020 (Table 1), thirty-seven patients were out of contact, including 1 patient with G1 NETs, 6 with G2 NETs, 8 with G3 NETs and 22 with G3 NECs. Of the 113 patients in touch, there were 72 death due to tumor progression or burden, including 4 G1 NETs, 11 G2 NETs, 15 G3 NETs and 42 G3 NECs. The estimated 3-year OS for the entire cohort with MH-NENs secondary to GEPNENs was $45.6 \%$, with a MST of 31.9mons (95\% Cls: 24.8-39.0mons). The estimated 3-year OS for patients with G1 NETs, G2 NETs, G3 NETs and G3 NECs was 100\%, 79.4\%, 49.5\% and 20.7\%, with a MST of 64.6mons (95\% Cls: 53.8-75.4mons), 48.5mons (95\% Cls: 40.9-56.1mons), 32.2mons (95\% Cls: 23.241.2mons) and 21.5mons (95\% Cls: 20.9-22.1mons), respectively ( $P<0.001$; Fig.1). Further analysis among each new grading group indicated that survival of G1 NETs or G2 NETs was significantly better than that of $G 3$ NETs ( $P=0.013, P=0.037$; respectively) and G3 NECs $(P=0.001, P<0.001$; respectively). Patients with G3 NETs present notably longer survival time than those with G3 NECs $(P=0.012)$, while survival comparison between G1 NETs and G2 NETs wasn't statistically different $(P=0.131)$.

Moreover, the estimated 3-year OS and MST of MH-NENs originated from gastrointestinal tract was $53.6 \%$ and 37.1 mons (95\% Cls: $30.3-43.9$ mons), which was significantly better than $35.0 \%$ and 26.4 mons (95\% Cls: 21.2-31.6mons) of those secondary to pancreas ( $P=0.012$; Fig.2). MH-NENs diagnosed synchronously with the primary lesions showed a much worse survival than metachronous tumors [36.3\% vs. 61.0\%; 24.4mons (95\% Cls: 15.2-33.6mons) vs. 38.8mons (95\% Cls: 16.9-60.7mons); $P=0.001$; Fig.3]. The 3-year OS and MST of MH-NENs with extrahepatic metastatic disease was $22.3 \%$ and 21.9mons (95\% Cls: 19.7-24.1mons), compared statistically shorter than those without [54.4\%; 38.4mons (95\% Cls: 31.9-44.8mons); P=0.002; Fig.4].

\section{Prognostic factors of MH-NENs secondary to GEP-NENs}

According to our demonstration (Table 2), patients' sex (HR: 1.253; 95\% Cls: 0.877-3.013; P=0.647) and age (HR: 0.992; 95\% Cls: 0.436-1.354; $P=0.518)$, tumor largest diameter (HR: 1.011; 95\% Cls: 0.457-1.504; $\mathrm{P}=0.083$ ) and preoperative adjuvant therapy (HR: $1.231 ; 95 \% \mathrm{Cls}: 0.514-1.993 ; \mathrm{P}=0.102)$ was not notably associated with the survival of patients with $\mathrm{MH}-\mathrm{NEN}$ secondary to GEP-NENs. Moreover, tumor type (HR: 1.342; 95\% Cls: 0.539-2.492; $\mathrm{P}=0.047$ ), incidental diagnosis (HR: $1.645 ; 95 \%$ Cls: 0.636-3.078; $\mathrm{P}=0.015)$, multifocal lesions (HR: 1.412; 95\% Cls: 0.514-2.009; $\mathrm{P}=0.036)$, estimated liver involvement (HR: 1.662; 95\% Cls: 0.763-2.567; $P=0.028)$, surgical procedure (HR: 1.443; 95\% Cls: 0.623-2.354; $P=0.043$ ), postoperative adjuvant therapy (HR: 1.402; 95\% Cls: 0.537-2.114; $\mathrm{P}=0.035$ ), regional lymph node metastases (HR: 1.834; 95\% Cls: 0.863-2.942; $\mathrm{P}=0.042$ ), portal vein tumor thrombus (HR: $2.034 ; 95 \% \mathrm{Cls:}$ 1.112-3.142; $P=0.039$ ) and vascular invasion (HR: 1.952; 95\% Cls: 0.885-3.432; $P=0.017$ ) was only statistically significant on univariate analysis. On multivariable analysis, synchronous liver lesion (HR: 
2.012; 95\% Cls: 1.092-3.475; $P=0.014)$, pancreatic primary site (HR: 1.882; 95\% Cls: 1.003-3.825; $P=0.031), R 1 / R 2$ resection (HR: 1.764; 95\% Cls: 0.743-4.111; $P=0.007)$, extrahepatic metastatic disease (HR: 3.053; 95\% Cls: 1.473-5.082; $P=0.027$ ) and G3 NECs by WHO 2017 grading classification (HR: 4.234; $95 \%$ Cls: 1.984-6.763; $\mathrm{P}=0.003$ ) was each related to worse long-term survival of patients with $\mathrm{MH}-\mathrm{NENs}$ secondary to GEP-NENs.

\section{Discussion}

NENs are a group of heterogeneous tumors with distinct clinicopathological features and potentially malignant behaviors, which mostly originated from digestive system, especially the gastro-enteropancreatic site ${ }^{[1-4]}$. As we mentioned before, $\mathrm{H}-\mathrm{NEN}$ s are rather uncommon and MH-NENs secondary to GEP-NENs account for their majority $[6,7,9,10]$. With the studying goal of our research, we finally identified 150 patients with MH-NENs secondary to GEP-NENs, which had a slight male predominance with a median age of $56 \mathrm{yrs}$. (range 42-74yrs.) (Table 1). This demography was much close to the reports by some previous studies ${ }^{[26,27]}$. In our study, 64 MH-NENs were secondary to pancreas (42.7\%), 96 cases were diagnosed synchronously with the primary lesions (64\%), 127 cases showed over $50 \%$ estimated liver involvement (84.7\%) and 42 cases simultaneously present extrahepatic invasion at diagnosis (28\%). Xiang et al. reported in their research that $44.9 \%$ of MH-NENs originated from pancreas, $65.4 \%$ of cases present synchronous liver metastasis, $82.5 \%$ of cases had over $50 \%$ estimated liver involvement and $11.1 \%$ of cases present extrahepatic invasion ${ }^{[27]}$. As a result, our estimated 3-year OS for MH-NENs secondary to GEP-NENs was $45.6 \%$, which was a little worse than that of previous studies ${ }^{[26,27]}$, probably due to the stricter inclusive criteria that MH-NENs in the present study was just secondary to gastroentero-pancreatic system, rather than other metastatic sites of NENs.

What's more, with an obviously rising incidence in the past decades, a uniform pathological classification for NENs has been lacking, which substantially disabled physicians to stratify patients into prognostic groups and to guide their managements ${ }^{[5-7]}$. In 2010, WHO defined the entire group of this disease as NENs and divided them into G1 NETs, G2 NETs and "G3 NECs" based on mitotic rate and Ki-67 proliferative index ${ }^{[13]}$. The WHO 2010 grading classification for NENs was practical, whose clinical value has been validated by subsequent studies ${ }^{[18-21]}$. However, recent studies have focused on the heterogeneity of WHO 2010 "G3 NECs" group, which might consist of morphologically well-differentiated NETs with a high proliferative rate and true poorly-differentiated NECs with small-cell or large-cell features for NENs originated both from pancreas ${ }^{[15,16,25,28]}$ and gastrointestinal tract ${ }^{[29-33]}$. In 2017 , based on some established research results on histopathologic criteria to better predict the tumor's grade and biological behaviors, WHO redefined GEP-NENs into well-differentiated tumors of G1 NETs, G2 NETs, G3 NETs and poorly-differentiated carcinomas of G3 NECs referring mainly to both morphological differentiation and grading upon proliferation rate ${ }^{[22]}$.

Till now, there isn't a specific grading system for H-NENs (either PH-NENs or MH-NENs) due to the rarity of their epidemiology or the heterogeneity of original tumors. Considering the homogeneity of metastasis 
lessons and primary tumors, WHO grading classifications originally proposed for GEP-NENs have also been introduced to MH-NENs secondary to GEP-NENs ${ }^{[13,14,22]}$. Using the WHO 2010 grading classification, Lv et al. reported the percentage of G1, G2 and G3 among patients with H-NENs were respectively $4.94 \%, 25.93 \%$ and $69.13 \%$, with a separate MST of 40.82 mons, 51.87 mons and 33.80 mons [26]. There was significant difference on the OS when comparing G1/G2 NETs with "G3 NECs" $(P=0.011)$, while survival difference between G1 NETs and G2 NETs was not statistically significant $(P>0.05)[26]$. Moreover, when analyzing the clinicopathological features of 112 patients with MH-NENs originating from digestive tract, Jiao et al. retrospectively enrolled 3 patients with WHO 2010 G1 NETs, 18 with G2 NETs and 91 with "G3 NECs" [34]. Interestingly, Jiao et al. identified 23 cases with G3 NETs in their "G3 NECs" category, which were morphologically well-differentiated with mean Ki-67 proliferative index of $44 \%$ (range $25 \%-60 \%$ ) ${ }^{[34]}$. They also demonstrated that the MST of G3 NETs for patients with MH-NENs originating from digestive tract was 24.0 mons, which was notably longer than 8.0 mons of G3 NECs group $(P<0.01)^{[34]}$.

The clinical value of the new WHO 2017 grading classification has not been rigorously validated before for patients with MH-NENs secondary to GEP-NENs. In the present study, in the light of the new WHO 2017 grading classification, we distributed all eligible patients into 4 groups for the first time, in which 10 patients with G1 NETs, 26 with G2 NETs, 33 with G3 NETs and 81 with G3 NECs were respectively identified. According to our analysis, significant differences between G1/G2/G3 NETs and G3 NECs were detected (Table 1), such as those of age at diagnosis $(P=0.041)$, synchronous liver lesion $(P=0.032)$, incidental diagnosis $(P=0.014)$, tumor largest diameter $(P=0.047)$, vascular invasion $(P=0.017)$ and extrahepatic metastatic disease $(P=0.029)$, which have resulted in the different survivals among each WHO 2017 grading group ( $P<0.001$; Fig. 1). Especially, survival of G3 NETs was notably better than those with G3 NECs $(P=0.012)$, but much worse than that of $G 1$ and $G 2$ NETs $(P=0.013, P=0.037$; respectively), whereas survival of G3 NECs was statistically worse than that of G1/G2/G3 NETs ( $P=$ $0.001, P<0.001, P=0.012$; respectively). Finally, we demonstrated that synchronous liver lesion, primary tumor site, surgical margin, extrahepatic metastatic disease and WHO 2017 grading classification were effective independent predictors of OS for patients with MH-NENs secondary to GEP-NENs (Table 2). Our analyses were essentially in agreement with the results by previous studies [27, 34].

Although we firstly evaluated the prognostic relevance of this novel WHO 2017 grading classification for patients with MH-NENs secondary to GEP-NENs, we still acknowledged the limitations of our study. First of all, we confined MH-NENs to those only secondary to GEP-NENs, which will be of uncertain value for MH-NENs originated from other metastasis sites of NENs, such as lung, adrenal gland, etc. Then, the study population was relatively small, especially those with G1 or G2 NETs, which may affect the accuracy of their survival analysis. Thirdly, because the time span of our study was too long, the detailed data of each patient's medical therapy was rather difficult to be collected, whose clinical effect would not be reflected accurately. Finally, the retrospective nature of the study prevented us from obtaining some detailed information, such as adjuvant therapy and tumor recurrence, which still needs to be further discussed. 


\section{Conclusion}

In conclusion, by applying the new WHO 2017 grading classification, we analyzed the clinical features and long-term survivals of patients with MH-NENs secondary to GEP-NENs. We found that G3 NECs behaved more aggressively than G1/G2/G3 NETs. Furthermore, we confirmed that synchronous liver lesion, pancreatic primary site, R1/R2 resection, extrahepatic metastatic disease and G3 NECs category were significantly associated with worse OS of patients with MH-NENs secondary to GEP-NENs, which clinically validated the prognostic value of this new WHO 2017 grading classification. In a word, our demonstration indicated that the WHO 2017 grading classification could well stratify MH-NENs secondary to GEP-NENs into prognostic groups and supported its wide use in clinical practice.

\section{Abbreviations}

NENs: Neuroendocrine neoplasms; GEP-NENs:gastro-entero-pancreatic neuroendocrine neoplasms; $\mathrm{H}$ NENs:hepatic neuroendocrine neoplasms; PH-NEN:primary hepatic neuroendocrine neoplasms; $\mathrm{MH}-$ NENs:metastatic hepatic neuroendocrine neoplasms; WHO:World Health Organization; G:Grading; NETs:neuroendocrine tumors; NECs:neuroendocrine carcinomas; HPFs:high power fields; OS:Overall survival; MST:median survival time; HR:hazard ratio; Cls:confidence intervals; K-M:Kaplan-Meier;

\section{Declarations}

\section{Ethics approval and consent to participate}

Our retrospective research was approved by the local ethics committee, which was in accordance with the general principles of the Helsinki Declaration.

\section{Consent for publication}

The written informed consent was acquired on admission from all patients for their information to be used for studying purpose.

\section{Availability of data and materials}

The data and materials of our present research couldn't be shared at this time as the data and materials also formed part of an ongoing study, while they could be available in the near future from the corresponding author upon request after the accomplishment of our ongoing study.

\section{Conflicts of Interest}

We declared that we had no conflict of interest among the authors. 


\section{Funding}

We declared that we didn't have any sponsorship.

\section{Authors' contributions}

In this paper, YM. contributed to this work as first author; ZY. and XB. contributed equally as senior author. $Z Y$. and $X B$. designed the research and approved the final manuscript; YM. extracted the data and wrote the manuscript; ZY. and XB. corrected the manuscript; ZL. made the tables and figures; HS.Z. and KN.W. carried out the reference review and statistical analysis; TB.L. and LX.B. had important intelligent contributions to the manuscript. All authors red and approved the final manuscript.

\section{Acknowledgement}

Not applicable.

\section{References}

1. Kloppel G, Perren A, Heitz PU. The gastroenteropancreatic neuroendocrine cell system and its tumors: the WHO classification. Ann N Y Acad Sci. 2004; 1014:13-27.

2. Kloppel G. Classification and pathology of gastroenteropancreatic neuroendocrine neoplasms. Endocr Relat Cancer. 2011; 18 Suppl 1: S1-S16.

3. Dasari A, Mehta K, Byers LA, Sorbye H, Yao JC. Comparative study of lung and extrapulmonary poorly differentiated neuroendocrine carcinomas: A SEER database analysis of 162,983 cases. Cancer. 2018; 124:807-815.

4. Turaga KK, Kvols LK. Recent progress in the understanding, diagnosis, and treatment of gastroenteropancreatic neuroendocrine tumors. CA Cancer J Clin. 2011; 61:113-132.

5. Yao JC, Hassan M, Phan A, Dagohoy C, Leary C, Mares JE, et al. One hundred years after "carcinoid": epidemiology of and prognostic factors for neuroendocrine tumors in 35,825 cases in the United States. J Clin Oncol. 2008; 26:3063-3072.

6. Garcia-Carbonero R, Capdevila J, Crespo-Herrero G, Díaz-Pérez JA, Martínez Del Prado MP, Alonso Orduña $\mathrm{V}$, et al. Incidence, patterns of care and prognostic factors for outcome of gastroenteropancreatic neuroendocrine tumors (GEP-NETs): results from the National Cancer Registry of Spain (RGETNE). Ann Oncol. 2010; 21:1794-1803.

7. Dasari A, Shen C, Halperin D, Zhao B, Zhou S, Xu Y, et al. Trends in the Incidence, Prevalence, and Survival Outcomes in Patients With Neuroendocrine Tumors in the United States. JAMA Oncol. 2017; 3:1335-1342. 
8. Korse CM, Taal BG, van Velthuysen ML, Visser O. Incidence and survival of neuroendocrine tumours in the Netherlands according to histological grade: experience of two decades of cancer registry. Eur J Cancer. 2013; 49:1975-1983.

9. Lepage C, Bouvier AM, Phelip JM, Hatem C, Vernet C, Faivre J. Incidence and management of malignant digestive endocrine tumours in a well defined French population. Gut. 2004; 53:549-553.

10. Parkash O, Ayub A, Naeem B, Najam S, Ahmed Z, Jafri W, et al. Primary Hepatic Carcinoid Tumor with Poor Outcome. J Coll Physicians Surg Pak. 2016; 26:227-229.

11. Kulke MH, Shah MH, Benson AB 3rd, Bergsland E, Berlin JD, Blaszkowsky LS, et al. Neuroendocrine tumors, version 1.2015. J Natl Compr Canc Netw. 2015; 13:78-108.

12. Frilling A, Clift AK. Therapeutic strategies for neuroendocrine liver metastases. Cancer. 2015; 121:1172-1186.

13. Rindi G, Klöppel G, Alhman H, Caplin M, Couvelard A, de Herder WW, et al. TNM staging of foregut (neuro) endocrine tumors: a consensus proposal including a grading system. Virchows Arch. 2006; 449:395-401.

14. Rindi G, Arnold R, Bosman FT, Capella C, Klimstra DS, Kloppel G, et al. Nomenclature and classification of neuroendocrine neoplasms of the digestive system. In: Bosman FT, Carneiro F, Hruban RH, Theise ND. WHO classification of tumours of the digestive system, 4th ed. Lyon: International Agency for Research on cancer; 2010: 13-40.

15. Sorbye H, Welin S, Langer SW, Vestermark LW, Holt N, Osterlund P, et al. Predictive and prognostic factors for treatment and survival in 305 patients with advanced gastrointestinal neuroendocrine carcinoma (WHO G3): the NORDIC NEC study. Ann Oncol. 2013; 24:152-160.

16. Basturk O, Yang Z, Tang LH, Hruban RH, Adsay V, McCall CM, et al. The high-grade (WHO G3) pancreatic neuroendocrine tumor category is morphologically and biologically heterogenous and includes both well differentiated and poorly differentiated neoplasms. Am J Surg Pathol. 2015; 39:683-690.

17. Cavalcanti MS, Gönen M, Klimstra DS. The ENETS/WHO grading system for neuroendocrine neoplasms of the gastroenteropancreatic system: a review of the current state, limitations and proposals for modifications. Int J Endocr Oncol. 2016; 3:203-219.

18. Panzuto F, Boninsegna L, Fazio N, Campana D, Brizzi MP, Capurso G, et al. Metastatic and locally advanced pancreatic endocrine carcinomas: analysis of factors associated with disease progression. J Clin Oncol. 2011; 29:2372-2377.

19. Dolcetta-Capuzzo A, Villa V, Albarello L, Franchi GM, Gemma M, Scavini M, et al. Gastroenteric neuroendocrine neoplasms classification: comparison of prognostic models. Cancer. 2013; 119:3644.

20. Yang M, Tian BL, Zhang Y, Su AP, Yue PJ, Xu S, et al. Evaluation of the World Health Organization 2010 Grading System in surgical outcome and prognosis of pancreatic neuroendocrine tumors. Pancreas. 2014; 43:1003-1008. 
21. Yang $M$, Ke NW, Zeng L, Zhang $Y$, Tan CL, Zhang H, et al. Survival Analyses for Patients With Surgically Resected Pancreatic Neuroendocrine Tumors by World Health Organization 2010 Grading Classifications and American Joint Committee on Cancer 2010 Staging Systems. Medicine (Baltimore). 2015; 94: e2156.

22. Klöppel G, Couvelard A, Hruban RH, Klimstra DS, Komminoth P, Osamura RY, Perren A, Rindi G. Tumours of the endocrine pancreas. In: Lloyd RV, Osamura RY, Klöppel G, Rosai J, eds. WHO Classification of the Tumours of Endocrine Organs, 4th ed. Lyon, France: International Agency for Research on Cancer; 2017:175-207.

23. Crawley FP. The Limits of the Declaration of Helsinki. In: Address to Scientific Session. World Medical Association General Assembly: Helsinki, 2012.

24. Yang M, Zhang Y, Zeng L, Ke NW, Tan CL, Tian BL, et al. Prognostic Validity of the American Joint Committee on Cancer Eighth Edition TNM Staging System for Surgically Treated and WellDifferentiated Pancreatic Neuroendocrine Tumors: A Comprehensive Analysis of 254 Consecutive Patients From a Large Chinese Institution. Pancreas. 2019; 48:613-621.

25. Yang M, Zhang Y, Zeng L, Ke NW, Tan CL, Tian BL, et al. Survivals of patients with surgically treated and High-grade pancreatic neuroendocrine carcinomas: A comparative study between two American Joint Committee on Cancer $8^{\text {th }}$ tumor-node-metastasis staging systems. Eur J Surg Oncol. 2019; 45:1054-1060.

26. Lv Y, Huang C, Xu HZ, Han X, Zhang L, Mao WL, et al. Clinicopathological Characteristics of the primary and metastatic Hepatic Neuroendocrine Tumors and the relevant Prognosis-Related Factors: A Retrospective Study of 81 Cases in a Single Chinese Center. J Cancer. 2018; 9:479-487.

27. Xiang JX, Zhang XF, Beal EW, Weiss M, Aldrighetti L, Poultsides GA, et al. Hepatic Resection for Nonfunctional Neuroendocrine Liver Metastasis: Does the Presence of Unresected Primary Tumor or Extrahepatic Metastatic Disease Matter? Ann Surg Oncol. 2018; 25:3928-3935.

28. Deng BY, Yang M, Wen JY, Hou SZ, Chen Y, Tian BL, et al. Survivals of patients with pancreatic neuroendocrine carcinomas: An in-depth analysis by the American Joint Committee on Cancer 8th tumor-node-metastasis staging manual. Medicine (Baltimore). 2020; 99: e18736.

29. Velayoudom-Cephise FL, Duvillard P, Foucan L, Hadoux J, Chougnet CN, Leboulleux S, et al. Are G3 ENETS neuroendocrine neoplasms heterogeneous? Endocr Relat Cancer. 2013; 20:649-657.

30. Heetfeld M, Chougnet CN, Olsen IH, Rinke A, Borbath I, Crespo G, et al. Characteristics and treatment of patients with G3 gastroenteropancreatic neuroendocrine neoplasms. Endocr Relat Cancer. 2015; 22:657-664.

31. Kidd M, Modlin I, Oberg K. Towards a new classification of gastroenteropancreatic neuroendocrine neoplasms. Nat Rev Clin Oncol. 2016; 13:691-705.

32. Milione M, Maisonneuve P, Spada F, Pellegrinelli A, Spaggiari P, Albarello L, et al. The clinicopathologic heterogeneity of grade 3 gastroenteropancreatic neuroendocrine neoplasms: morphological differentiation and proliferation identify different prognostic categories. Neuroendocrinology. 2017; 104:85-93. 
33. Yin XN, Shen $C Y$, Yin YQ, Chen HJ, Chen HN, Yin Y, et al. Prognoses in patients with primary gastrointestinal neuroendocrine neoplasms based on the proposed new classification scheme. AsiaPac J Clin Oncol. 2018;14: e37-e44.

34. Jiao XX, Wang ZD, Zhang TF, Zhang LF, Ma W, Zhou Li. Clinicopathological features $\$ diagnosis and treatment of primary and metastatic hepatic neuroendocrine neoplasm originating from digestive tract. Chin J Dig. 2019; 39:752-758.

\section{Tables}

Table 1. Clinical features of MH-NENs secondary to GEP-NENs according to the WHO 2017 grading classification $^{A}$ 


\begin{tabular}{|c|c|c|c|c|c|c|}
\hline Characteristics & $\begin{array}{l}\text { NET G1 } \\
(\mathrm{N}=10)\end{array}$ & $\begin{array}{l}\text { NET G2 } \\
(\mathrm{N}=26)\end{array}$ & $\begin{array}{l}\text { NET G3 } \\
(\mathrm{N}=33)\end{array}$ & $\begin{array}{l}\text { NEC G3 } \\
(\mathrm{N}=81)\end{array}$ & $\begin{array}{l}\text { All } \\
(N=150)\end{array}$ & $P$ value ${ }^{B}$ \\
\hline \multicolumn{4}{|l|}{ Sex, male, n (\%) } & \multicolumn{3}{|l|}{0.154} \\
\hline & $6(60.0 \%)$ & $15(57.7 \%)$ & $19(57.6 \%)$ & $50(61.7 \%)$ & $90(60.0 \%)$ & \\
\hline \multicolumn{4}{|c|}{ Age at diagnosis, yrs. } & \multicolumn{2}{|l|}{0.041} & \\
\hline Median (Range) & $49(42-59)$ & $53(44-64)$ & $55(42-69)$ & $59(45-74)$ & $56(42-74)$ & \\
\hline \multicolumn{4}{|c|}{ Synchronous liver lesion } & \multicolumn{2}{|l|}{0.032} & \\
\hline & $5(50.0 \%)$ & $15(57.7 \%)$ & $20(60.6 \%)$ & $56(69.1 \%)$ & $96(64.0 \%)$ & \\
\hline \multicolumn{2}{|c|}{ Primary tumor site } & \multicolumn{5}{|c|}{0.166} \\
\hline Stomach & $2(20.0 \%)$ & $5(19.2 \%)$ & $6(18.2 \%)$ & $17(20.9 \%)$ & $30(20.0 \%)$ & \\
\hline Small intestine & $1(10.0 \%)$ & $3(11.5 \%)$ & $4(12.1 \%)$ & $4(4.9 \%)$ & $12(8.0 \%)$ & \\
\hline Large intestine & $3(30.0 \%)$ & $8(30.8 \%)$ & $8(24.2 \%)$ & $25(30.8 \%)$ & $44(29.3 \%)$ & \\
\hline Pancreas & $4(40.0 \%)$ & $10(38.5 \%)$ & $15(45.5 \%)$ & $35(43.4 \%)$ & $64(42.7 \%)$ & \\
\hline \multicolumn{2}{|c|}{ Non-functional tumor } & \multicolumn{5}{|c|}{0.209} \\
\hline & $6(60.0 \%)$ & $13(50.0 \%)$ & $18(54.5 \%)$ & $47(58.0 \%)$ & $84(56.0 \%)$ & \\
\hline \multicolumn{2}{|c|}{ Incidental diagnosis } & \multicolumn{5}{|c|}{0.014} \\
\hline & $3(30.0 \%)$ & $6(23.1 \%)$ & $10(30.3 \%)$ & $29(35.8 \%)$ & $48(32.0 \%)$ & \\
\hline \multicolumn{2}{|c|}{ Multifocal lesions ${ }^{C}$} & & \multicolumn{4}{|c|}{0.512} \\
\hline & $8(80.0 \%)$ & $17(65.4 \%)$ & $25(75.7 \%)$ & $60(74.1 \%)$ & $110(73.3 \%)$ & \\
\hline \multicolumn{2}{|l|}{ Tumor diameter ${ }^{D}$} & \multicolumn{5}{|c|}{0.047} \\
\hline Median (Range) & $8(2-9)$ & $9(2-15)$ & $11(3-17)$ & $15(4-24)$ & $13(2-24)$ & \\
\hline \multicolumn{2}{|l|}{ Tumor location } & & & \multicolumn{2}{|l|}{0.634} & \\
\hline Left half liver & $1(10.0 \%)$ & $4(15.4 \%)$ & $5(15.1 \%)$ & $7(8.7 \%)$ & $17(11.3 \%)$ & \\
\hline Right half liver & $3(30.0 \%)$ & $6(23.1 \%)$ & $9(27.3 \%)$ & $23(28.4 \%)$ & $41(27.4 \%)$ & \\
\hline Both & $6(60.0 \%)$ & $16(61.5 \%)$ & $19(57.6 \%)$ & $51(62.9 \%)$ & $92(61.3 \%)$ & \\
\hline \multicolumn{2}{|c|}{ Estimated liver involvement } & & & \multicolumn{2}{|c|}{0.591} & \\
\hline$\leq 50 \%$ & $3(30.0 \%)$ & $7(26.9 \%)$ & $8(24.2 \%)$ & $20(24.7 \%)$ & $40(26.7 \%)$ & \\
\hline \multicolumn{2}{|c|}{ Surgical procedure } & \multicolumn{5}{|c|}{0.093} \\
\hline Resection & $3(30.0 \%)$ & $10(38.5 \%)$ & $12(36.4 \%)$ & $32(39.5 \%)$ & $57(38.0 \%)$ & \\
\hline
\end{tabular}




\begin{tabular}{|c|c|c|c|c|c|}
\hline Ablation & $3(30.0 \%)$ & $6(23.1 \%)$ & $7(21.1 \%)$ & $14(17.3 \%)$ & $30(20.0 \%)$ \\
\hline Both & $2(20.0 \%)$ & $6(23.1 \%)$ & $9(27.3 \%)$ & $24(29.6 \%)$ & $41(27.3 \%)$ \\
\hline Others ${ }^{\mathrm{E}}$ & $2(20.0 \%)$ & $4(15.3 \%)$ & $5(15.2 \%)$ & $11(13.6 \%)$ & $22(14.7 \%)$ \\
\hline \multicolumn{4}{|c|}{ Surgical margin } & \multicolumn{2}{|l|}{0.135} \\
\hline $\mathrm{RO}^{\mathrm{F}}$ & $4(40.0 \%)$ & $11(42.3 \%)$ & $12(36.4 \%)$ & $30(37.1 \%)$ & $57(38.0 \%)$ \\
\hline \multicolumn{4}{|c|}{ Preoperative adjuvant therapy } & \multicolumn{2}{|c|}{0.542} \\
\hline & $6(60.0 \%)$ & $16(61.6 \%)$ & $22(66.7 \%)$ & $49(60.5 \%)$ & $93(62.0 \%)$ \\
\hline \multicolumn{4}{|c|}{ Postoperative adjuvant therapy } & \multicolumn{2}{|c|}{0.436} \\
\hline & $8(80.0 \%)$ & $20(77.0 \%)$ & $28(84.8 \%)$ & $72(88.9 \%)$ & $128(85.3 \%)$ \\
\hline \multicolumn{3}{|c|}{ Regional lymph node metastases } & \multicolumn{3}{|c|}{0.401} \\
\hline & $3(30.0 \%)$ & $10(38.5 \%)$ & $12(36.4 \%)$ & $32(39.5 \%)$ & $57(38.0 \%)$ \\
\hline \multicolumn{3}{|c|}{ Portal vein tumor thrombus } & \multicolumn{3}{|c|}{0.803} \\
\hline & $4(40.0 \%)$ & $12(46.2 \%)$ & $14(42.4 \%)$ & $35(43.2 \%)$ & $65(43.3 \%)$ \\
\hline \multicolumn{3}{|c|}{ Vascular invasion } & \multicolumn{3}{|c|}{0.017} \\
\hline & $1(10.0 \%)$ & $3(11.5 \%)$ & $5(15.2 \%)$ & $18(22.2 \%)$ & $27(18.8 \%)$ \\
\hline \multicolumn{3}{|c|}{ Extrahepatic metastatic disease } & \multicolumn{3}{|c|}{0.029} \\
\hline & $2(20.0 \%)$ & $5(19.2 \%)$ & $8(24.2 \%)$ & $27(33.3 \%)$ & $42(28.0 \%)$ \\
\hline \multicolumn{3}{|c|}{ Out of contact } & \multicolumn{3}{|c|}{ NA } \\
\hline & $1(10.0 \%)$ & $6(23.1 \%)$ & $8(24.2 \%)$ & $22(27.2 \%)$ & $37(24.7 \%)$ \\
\hline \multicolumn{3}{|c|}{ Dead at follow-up } & \multicolumn{3}{|c|}{ NA } \\
\hline & $4(44.4 \%)$ & $11(55.0 \%)$ & $15(60.0 \%)$ & $42(71.2 \%)$ & $72(63.7 \%)$ \\
\hline \multicolumn{3}{|c|}{ Estimated 3-year OS } & \multicolumn{3}{|c|}{$<0.001$} \\
\hline & $100 \%$ & $79.4 \%$ & $49.5 \%$ & $20.7 \%$ & $45.6 \%$ \\
\hline \multirow{2}{*}{\multicolumn{2}{|c|}{ MST, mons. }} & & \multicolumn{3}{|c|}{$<0.001$} \\
\hline & 64.6 & 48.5 & 32.2 & 21.5 & 31.9 \\
\hline
\end{tabular}

A: These characteristics of MH-NENs secondary to GEP-NENs were mainly based on the preoperative imaging examinations, intraoperative surgical findings and postoperative pathological analysis.

B: Referring to the comparison between those with NET G1/G2/G3 and those with NEC G3 wherever possible. 

C: Referring to no less than 1 metastatic lesions in liver.
D: Referring to the sum of the largest diameter of all visible hepatic lesions.

E: Referring to some uncommon procedures, such as alcohol injection and liver transplantation, etc.

F: Referring to radical resections with both grossly and microscopically negative surgical margins.

Abbreviations: MH-NENs: metastatic hepatic neuroendocrine neoplasms; GEP-NENs: Gastro-enteropancreatic neuroendocrine neoplasms; WHO: World Health Organization; NET: neuroendocrine tumor; NEC: neuroendocrine carcinoma; G: grading; TNM: tumor-node-metastasis; AJCC: American Joint Committee On Cancer; NA: Not applicable; OS: overall survival; MST: median survival time.

Table 2. Univariate and multivariate analysis of factors with the OS of MH-NENs secondary to GEP-NENs using Cox Regression proportional hazard models. 


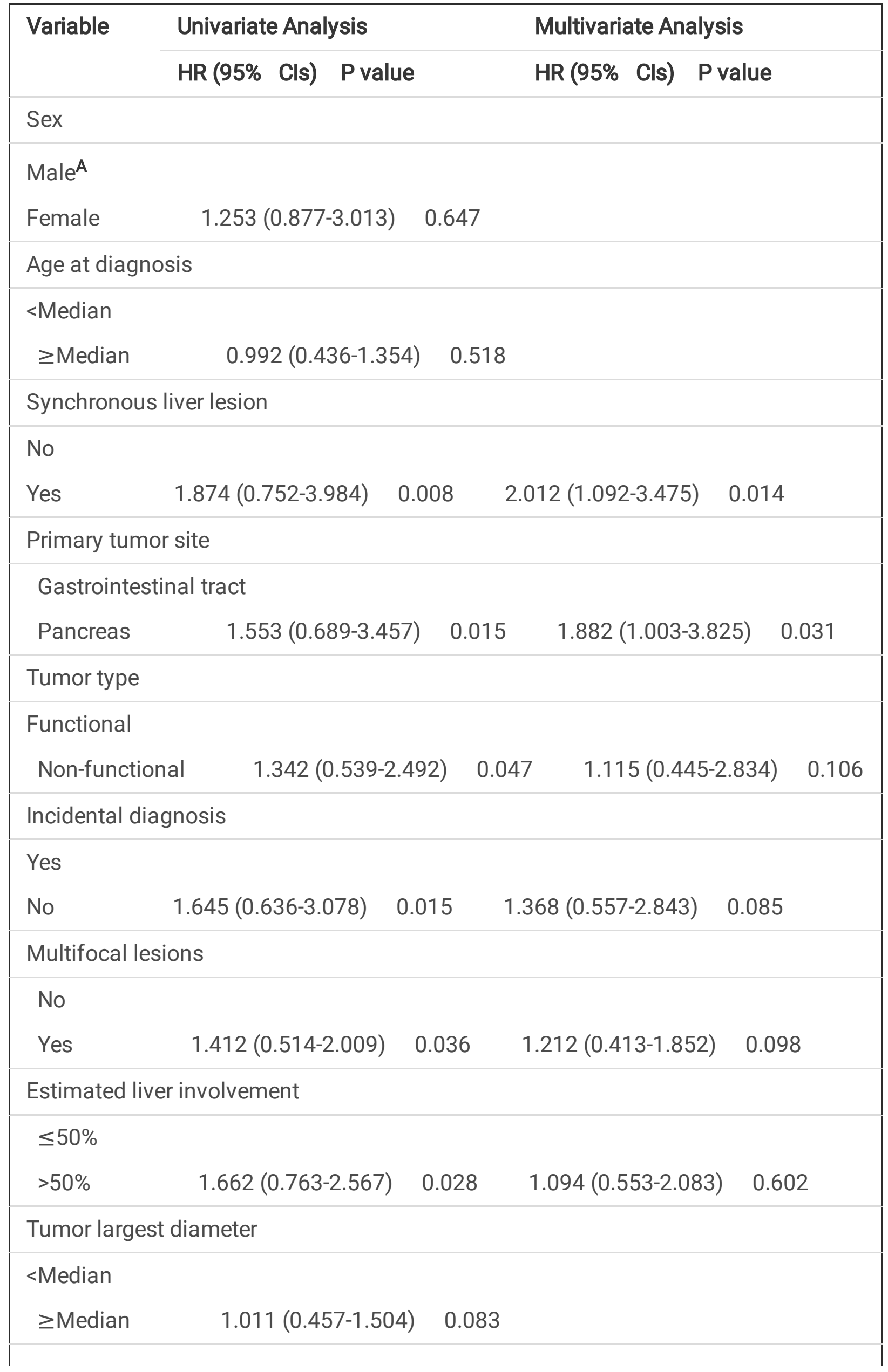




\begin{tabular}{|c|c|c|c|c|}
\hline \multicolumn{5}{|c|}{ Surgical procedure } \\
\hline \multicolumn{5}{|c|}{ Resection } \\
\hline Ablation & $1.443(0.623-2.354)$ & 0.043 & $0.942(0.325-2.012)$ & 0.114 \\
\hline \multicolumn{5}{|c|}{ Surgical margin } \\
\hline \multicolumn{5}{|l|}{ Ro } \\
\hline $\mathrm{R} 1 / \mathrm{R} 2$ & $2.135(0.994-4.413)$ & $<0.001$ & $1.764(0.743-4.111)$ & 0.007 \\
\hline \multicolumn{5}{|c|}{ Preoperative adjuvant therapy } \\
\hline \multicolumn{5}{|l|}{ Yes } \\
\hline No & $1.231(0.514-1.993)$ & 0.102 & & \\
\hline \multicolumn{5}{|c|}{ Postoperative adjuvant therapy } \\
\hline \multicolumn{5}{|l|}{ Yes } \\
\hline No & $1.402(0.537-2.114)$ & 0.035 & $1.132(0.446-1.764)$ & 0.236 \\
\hline \multicolumn{5}{|c|}{ Regional lymph node metastases } \\
\hline \multicolumn{5}{|l|}{ No } \\
\hline Yes & $1.834(0.863-2.942)$ & 0.042 & $1.435(0.567-2.715)$ & 0.157 \\
\hline \multicolumn{5}{|c|}{ Portal vein tumor thrombus } \\
\hline \multicolumn{5}{|l|}{ No } \\
\hline Yes & $2.034(1.112-3.142)$ & 0.039 & $1.573(0.894-2.064)$ & 0.159 \\
\hline \multicolumn{5}{|c|}{ Vascular invasion } \\
\hline \multicolumn{5}{|l|}{ No } \\
\hline Yes & $1.952(0.885-3.432)$ & 0.017 & $1.562(0.645-2.084)$ & 0.103 \\
\hline \multicolumn{5}{|c|}{ Extrahepatic metastatic disease } \\
\hline \multicolumn{5}{|l|}{ No } \\
\hline Yes & $2.143(1.249-3.985)$ & 0.002 & $3.053(1.473-5.082)$ & 0.027 \\
\hline \multicolumn{5}{|c|}{ Tumor grade by WHO 2017 classification } \\
\hline \multicolumn{5}{|c|}{ NET G1/G2/G3 } \\
\hline NEC G3 & $2.653(1.419-4.255)$ & $<0.001$ & $4.234(1.984-6.763)$ & 0.003 \\
\hline
\end{tabular}

A: The above one of related variable was regarded as a reference in Cox analysis. 
Abbreviations: OS: overall survival; MH-NENs: metastatic hepatic neuroendocrine neoplasms; GEP-NENs: Gastro-entero-pancreatic neuroendocrine neoplasms; HR: hazard ratio; Cls: confidence intervals; WHO: World Health Organization; NET: neuroendocrine tumors; NEC: neuroendocrine carcinoma; G: grading.

\section{Figures}

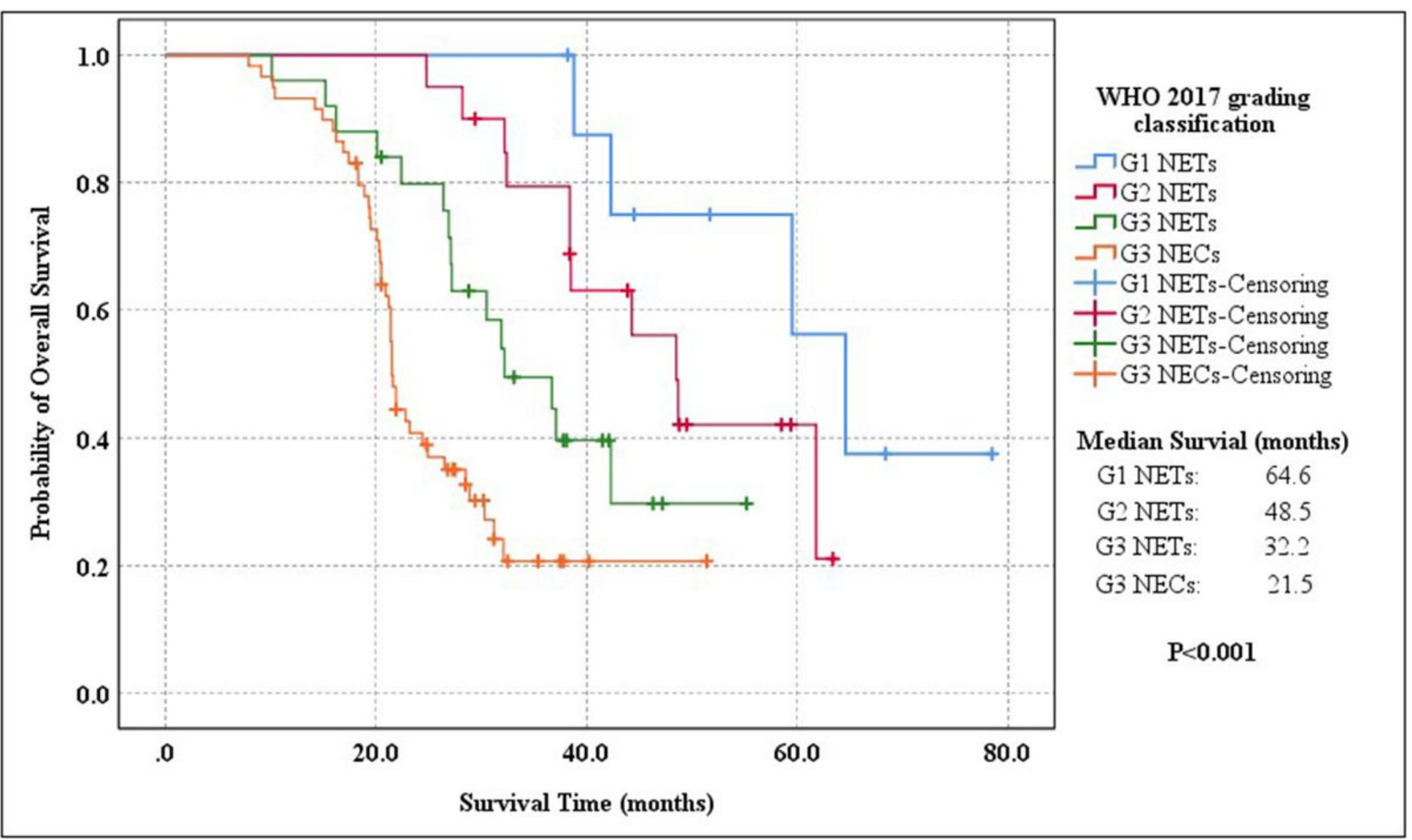

\section{Figure 1}

Kaplan-Meier estimates for the overall survival of metastatic hepatic neuroendocrine neoplasms secondary to gastro-entero-pancreatic site, according to World Health Organization 2017 grading classification proposed for gastro-entero-pancreatic neuroendocrine neoplasms. 


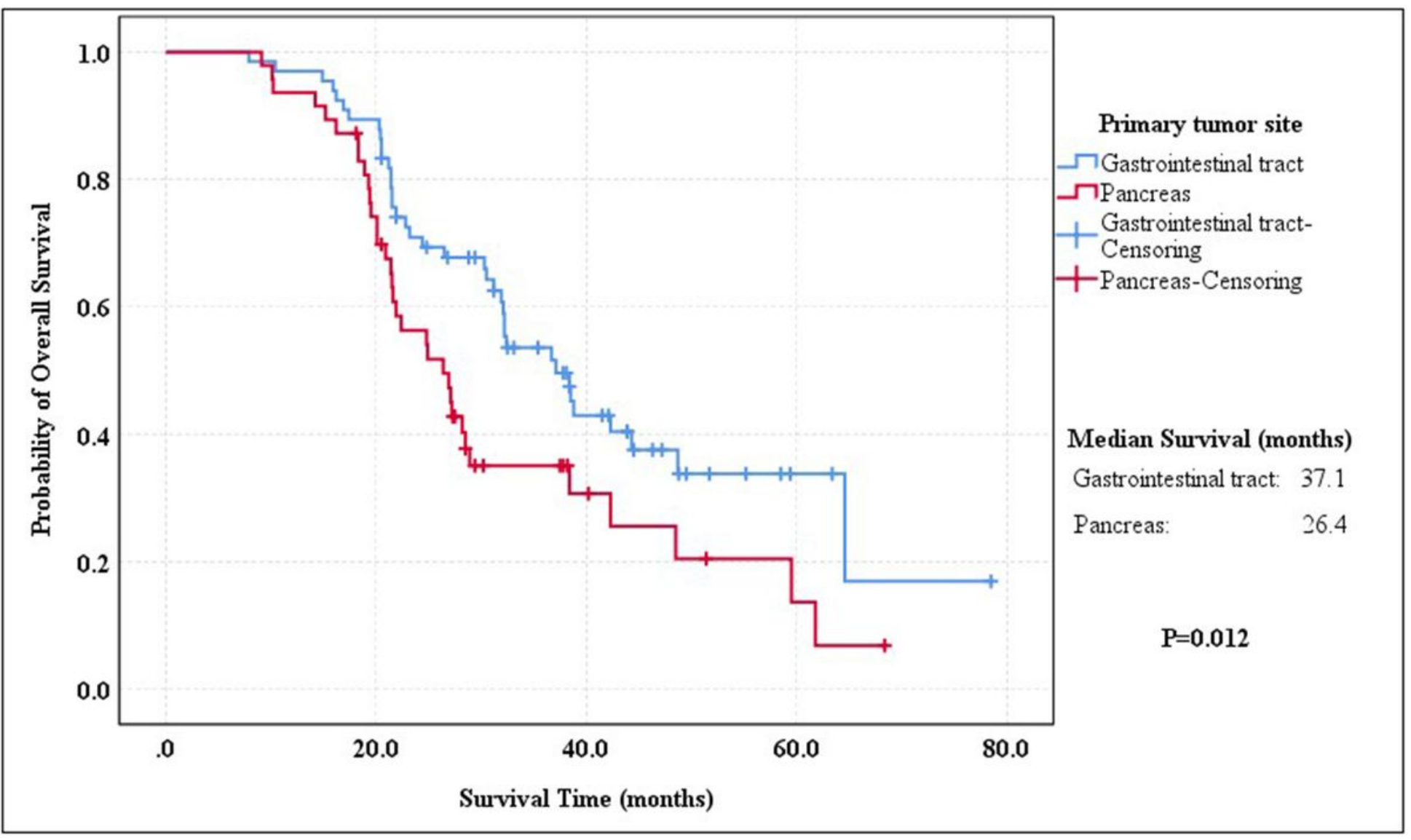

Figure 2

Kaplan-Meier estimates for the overall survival of metastatic hepatic neuroendocrine neoplasms secondary to gastro-entero-pancreatic site, according to primary tumor site. 


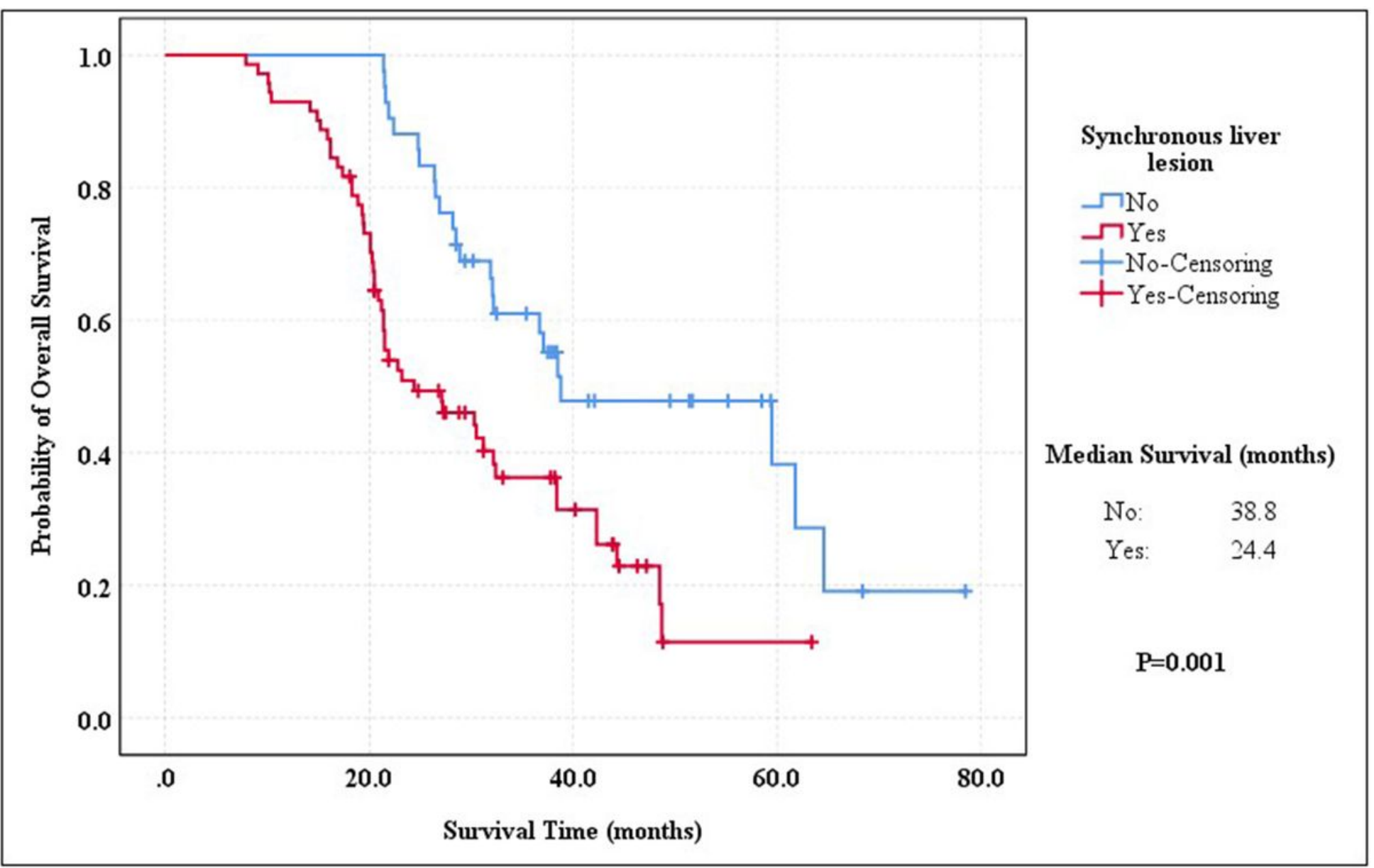

\section{Figure 3}

Kaplan-Meier estimates for the overall survival of metastatic hepatic neuroendocrine neoplasms secondary to gastro-entero-pancreatic site, according to synchronous liver lesion. 


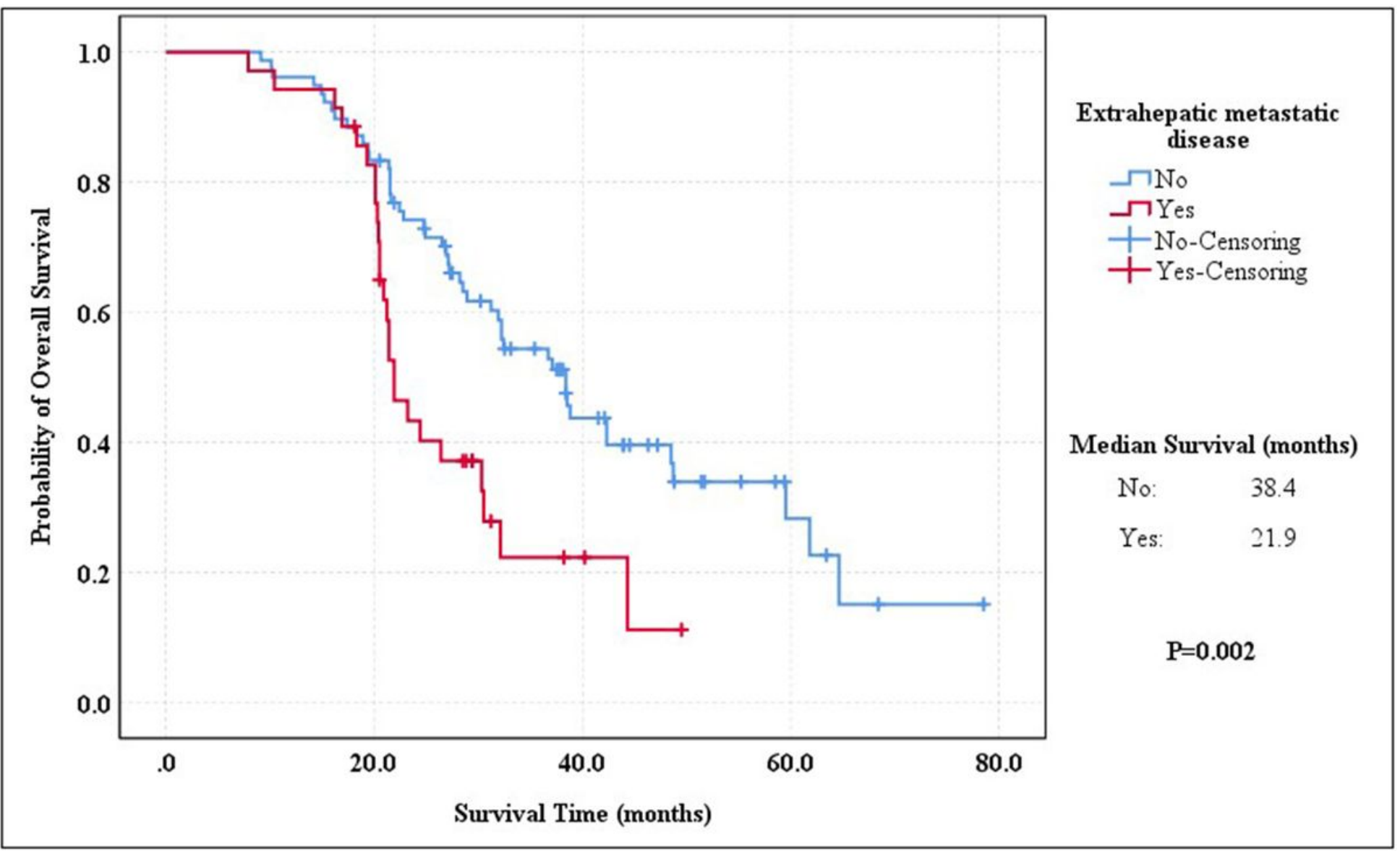

\section{Figure 4}

Kaplan-Meier estimates for the overall survival of metastatic hepatic neuroendocrine neoplasms secondary to gastro-entero-pancreatic site, according to extrahepatic metastatic disease. 